\title{
Interactions of Visinin-like Proteins with Phospho-inositides
}

\author{
Karl-Heinz Braunewell, ${ }^{A, B}$ Blessy Paul, ${ }^{C}$ Wassim Altarche-Xifro, ${ }^{A}$ Cornelia Noack, ${ }^{A}$ Kristian \\ Lange, ${ }^{A}$ and Andreas Hofmann ${ }^{C, D}$
}

ASignal Transduction Research Group, Neuroscience Research Center/Institute for

Neurophysiology, Charité Berlin, Germany.

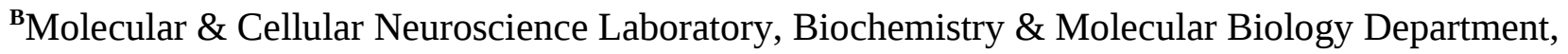
Southern Research Institute, Birmingham, AL 35205, USA.

'Structural Chemistry Program, Eskitis Institute for Cell \& Molecular Therapies, Griffith University, Brisbane, Qld 4111, Australia.

${ }^{\mathrm{D} C}$ Corresponding author. Email: a.hofmann@griffith.edu.au

Manuscript: p_16.doc, 21.06.09

Word count: 4728 
The subcellular membrane localisation of neuronal calcium sensor (NCS) proteins in living cells, such as VILIP-1 and VILIP-3, differs substantially. We have followed the hypothesis that the differential localisation might be due to specific binding capabilities of individual VILIPs for phosphatidylinositol phosphates (PIPs). Several highly conserved lysine residues in the N-terminal region could provide favourable electrostatic interactions.

Molecular modelling results support a binding site for phospho-inositides in the N-terminal area of VILIP-1, and the involvement of the conserved N-terminal lysine residues in binding the phosphoinositol head group. Experimentally, the binding of VILIP-1 to inositol derivatives was tested by a PIP strip assay, which showed the requirement of phosphorylation of the inositol group for the interaction of the protein with PIPs. Monolayer adsorption measurements showed a preference of VILIP-1 binding to $\mathrm{PI}(4,5) \mathrm{P}_{2}$ over $\mathrm{PI}(3,4,5) \mathrm{P}_{3}$. The co-localisation of VILIP-1 with $\mathrm{PI}(4,5) \mathrm{P}_{2}$ at the cell surface membrane in hippocampal neurons further supports the idea of direct interactions of VILIP-1 with PIPs in living cells. 


\section{Introduction}

The family of neuronal calcium sensor (NCS) proteins comprises five subfamilies, namely recoverins, frequenins, Visinin-like Proteins (VILIPs), GCAPs and KChlPs, which are involved in various signalling cascades in neuronal cells [1-3]. All members of the NCS protein family are EFhand proteins and share the typical feature of N-terminal myristoylation at the motif $\mathrm{M}-\mathrm{G}-\mathrm{X}_{3}-\mathrm{S}$, as well as the calcium-myristoyl switch. In response to elevated calcium levels, NCS proteins invoke the calcium-myristoyl switch which is the central mechanism of their involvement in cellular calcium signalling $[4 ; 5]$. It has been shown that membrane association of proteins by a myristoyl group alone is very weak and requires further stabilising interactions such as hydrogen bonds between the protein and membrane phospholipids or the involvement of another membraneassociated protein [6].

Protein-phospholipid interactions not only strengthen the membrane association but also play a role in targeting various proteins to different membrane domains [7; 8]. Since the lipid composition at the cell membrane is not uniform and micro domains rich in particular types of lipids and signalling molecules can be found, the preference of a protein for a certain region of the membrane can be determined by the type of phospholipid head group [9].

VILIPs show interesting differences in their subcellular localisation [10]. Burgoyne and co-workers demonstrated that myristoylated N-terminal peptides of hippocalcin, belonging to the VILIP subfamily, target to plasma membrane and Golgi [11]. VILIP-1 has been shown to localise to the plasma membrane, axonal and dendritic membranes, the Golgi, as well as clathrin-coated vesicles [12-14]. As to phospholipid compositions, the plasma membrane and the Golgi are rich in $\mathrm{PI}(4,5) \mathrm{P}_{2}$, and clathrin-coated vesicles are enriched in $\mathrm{PI}(4,5) \mathrm{P}_{2}$, as well as $\mathrm{PI}(3,4,5) \mathrm{P}_{3}[15]$.

The N-terminal region of hippocalcin has previously been implicated in binding of the protein to $\mathrm{PI}(4,5) \mathrm{P}_{2}$ [11]. At the molecular level, NCS-1, neurocalcin $\delta$ and hippocalcin, as well as VILIPs possess a cluster of basic residues in the very N-terminal region (VILIP-1: Lys3, Lys7, Lys32, Lys36). In VILIP-1, an extra lysine is present at position 28. Recoverin, in contrast, has a less 
positive electrostatic potential, and GCAP-2 possesses only two basic residues (Lys26, Lys27) in the N-terminal area. Structurally, a positively charged exposed N-terminal domain might act as anchor to the head groups of the membrane bilayer lipids and thus strengthen the protein-membrane association. Negatively charged lipids are mainly phosphatidylserine (PS) and phosphatidylinositol (PI) derivatives. While PS possesses a charge of -1 , PI derivatives may have charges of -3 to -7 depending on the degree of phosphorylation as well as external parameters such as local $\mathrm{pH}$ and effects of interacting proteins [16]. The positive electrostatic potential, as well as the membraneexposed conformation of the very N-terminal region of VILIPs support the idea of VILIP-PIP interactions rather than VILIP-PS interactions.

Our first goal in this study was to explore the hypothesis of phospho-inositide binding to VILIP-1 by a molecular modelling approach, using the previously generated homology models for VILIP-1. Secondly, we attempted to experimentally verify the postulated interaction of VILIP-1 with different PIP derivatives. Therefore, we have tested the VILIP-1-phospholipid interaction by examining the binding of myristoylated VILIP-1 to immobilised phospholipids. Adsorption of VILIP-1 to phospholipid monolayers was also tested using a Langmuir surface film balance, showing the preference of the protein for $\mathrm{PI}(4,5) \mathrm{P}_{2}$ over $\mathrm{PI}(3,4,5) \mathrm{P}_{3}$. Co-localisation studies in living cells support the notion of direct interactions between VILIP-1 and phospho-inositides.

\section{Results and Discussion}

\section{Ligand docking}

Based on the high similarity of VILIPs with neurocalcin $\delta$ and recoverin, we have recently generated homology models of VILIP-1 and VILIP-3 in the apo- and the calcium-bound forms [10; 17]. Automated docking of glyceryl-phosphatidyl inositol derivatives (GIPs) to the calcium-bound form of VILIP-1 was unsuccessful, most likely due to the spatial restrictions around the putative binding site in the homology model of VILIP-1 and the limitations of the automated approach (data 
not shown). We then modified the homology model by a minimal rotation of the N-terminal region against the core of the protein around the hinge between Thr20 and Glu21. This subtle conformational change allowed placement of GIP ligands in the putative binding site. The resulting complexes were used as starting models for molecular dynamics simulations. Molecular dynamics were simulated for $500 \mathrm{ps}$, and the plots of time-dependent rms deviations for the ligands, as well as for the protein backbone show that near-equilibrium states have been reached (Fig. 1).

\section{The N-terminal area of VILIPs in the calcium-bound conformation}

In the calcium-bound forms in the absence of ligand (apo-form), the N-terminal tail (residues 1-8) of VILIP-1 adopts an extended conformation and its axis is almost parallel to the axis of helix E1. The tail is placed roughly at a distance of $8 \AA$ from helix E1.

In the $\mathrm{GI}(4,5) \mathrm{P}_{2}$-bound form, one observes a movement of about $5 \AA$ of the $\mathrm{N}$-terminal tail towards helix E1 to accommodate the ligand. Notably, the overall orientation of the N-terminal tail with respect to the rest of the protein stays the same. With $\mathrm{GI}(3,4,5) \mathrm{P}_{3}$, in contrast, the protein seems to open up the ligand binding site by pulling the N-terminal tail away from helix E1. To accommodate $\mathrm{GI}(3,4,5) \mathrm{P}_{3}$, VILIP-1 undergoes a conformational change whereby the N-terminal tail is tilted away from the protein and the axes of EF1 and the tail are almost perpendicular. This is due to a shift of the N-terminal helix towards the protein and a drastic change in the conformation of Lys7 where the backbone-determining $\phi$ angle changes by about $180^{\circ}$.

\section{VILIP-PIP interactions}

Analysing the results from the molecular dynamics study, the orientation of VILIP-bound GI(4,5) $\mathrm{P}_{2}$ appears as the ligand sitting on top of the protein surface and all functional groups of the inositol ring are involved in ligand-protein interactions, except for 6-OH (Fig. 2). A total of four hydrogen bonds can be identified between Ser6, Lys7 and Lys28 of VILIP-1 and the ligand GI(4,5) $\mathrm{P}_{2}$. 
Additionally, a fifth hydrogen bond seems possible since the $\varepsilon$-amino group of Lys7 could easily adopt a conformation that is in bonding distance to the 5-phosphate of the ligand.

The situation is different with GI(3,4,5) $\mathrm{P}_{3}$ where the ligand slots "sideways" into the cleft opened between the N-terminal tail and helix E1. Here, all functional groups of the inositol ring are involved in interactions with the protein and only the 2-OH group remains unsaturated (Fig. 2). Lys36 recognises the 3-phosphate, Lys32 the 4-phosphate, and Ser6 interacts with the 5-phosphate.

\section{Adsorption to phospholipid monolayers}

To further test the hypothesis of VILIP-1 recognising phospho-inositides, the protein was subjected to phospholipid monolayer adsorption experiments using a Langmuir surface film balance. Protein is injected into the subphase of an equilibrated monolayer consisting of DMPS/DMPC (3:1), and the surface pressure $\pi$ will increase from its initial value $\pi_{0}$ upon binding of the protein to the monolayer. Over the time course of about 45 min, VILIP-1 in the absence of calcium elicits a very slow increase of the surface pressure $\left(\Delta \pi=2 \mathrm{mN} \mathrm{m}^{-1}\right)$ after a lag phase of about $15 \mathrm{~min}$ (Fig. 3). When testing monolayers that contain either $1 \% \mathrm{PI}(4,5) \mathrm{P}_{2}$ or $\mathrm{PI}(3,4,5) \mathrm{P}_{3}$, a sudden increase in surface pressure is observed, with a total pressure difference of $\Delta \pi=3.5 \mathrm{mN} \mathrm{m}^{-1}$ and $4.5 \mathrm{mN} \mathrm{m}^{-1}$ for $\mathrm{PI}(3,4,5) \mathrm{P}_{3}$ and $\mathrm{PI}(4,5) \mathrm{P}_{2}$, respectively. While the time course of the binding isotherm for $\mathrm{PI}(4,5) \mathrm{P}_{2}$ appears as a one-phase process, the isotherm obtained for $\mathrm{PI}(3,4,5) \mathrm{P}_{3}$ is reminiscent of a two-phase process.

These experiments demonstrate that even in the absence of calcium, VILIP-1 can interact with phospho-inositides embedded in a membrane monolayer. Taking into account the structural context of the proposed PIP binding site in the N-terminal region, it appears that membrane-anchored PIP can elicit a conformational change of VILIP-1 from its "closed" to an "open" state that allows access to the binding site. While this is believed to happen upon binding of calcium to the protein during the calcium-myristoyl switch, the current results suggest that a similar mechanism can be triggered by membrane-anchored PIP. The comparison of isotherms obtained with $\mathrm{PI}(4,5) \mathrm{P}_{2}$ and 
$\mathrm{PI}(3,4,5) \mathrm{P}_{3}$ further show that the interactions are due to specific recognition rather than chargeddriven adsorption. Firstly, the PIP-containing monolayers have been constructed with charge conservation, i.e. the PS component was slightly reduced in the case of the PIP-containing monolayers. Secondly, the binding of VILIP-1 was more pronounced in case of the $\mathrm{PI}(4,5) \mathrm{P}_{2}$ than in the $\mathrm{PI}(3,4,5) \mathrm{P}_{3}$ monolayer, arguing against an attraction of the protein due to the higher negative charge of $\mathrm{PI}(3,4,5) \mathrm{P}_{3}$.

\section{Phospholipid overlay assays}

In order to experimentally test our hypothesis that VILIP-1 is able to interact with phospholipids, we examined binding of myristoylated VILIP-1 to various phospholipids in a PIP-strip assay. VILIP-1 was found to bind to phospho-inositides and phosphatidic acid, but not to phosphatidylserine, -cholin, or -ethanolamine (Fig. 4). Notably, the protein did not bind to phosphoinositol lacking the ring phosphorylations. Non-myristoylated GST-VILIP-1 was used as a control and does not show any significant binding. While this might indicate that $\mathrm{N}$-terminal myristoylation of VILIP-1 is a prerequisite for VILIP-1 -membrane interactions, it is more likely that the binding of the fusion protein is hampered by the presence of the GST-tag. Despite the eventual hindering effects of the GST moiety of GST-VILIP-1 as to access to the N-terminal region, this control excludes the existence of an unspecific binding site on the surface of VILIP-1 under these circumstances.

When compared to the known binding of hippocalcin, which is very closely related to VILIP-3, some major differences in binding capability became obvious. Whereas hippocalcin is able to bind to phosphatidylinositol, VILIP-1 does not show any binding ability towards this phospholipid. In contrast, VILIP-1 strongly interacts with highly phosphorylated PI derivatives including $\mathrm{PI}(3,4) \mathrm{P}_{2}$, $\mathrm{PI}(3,5) \mathrm{P}_{2}, \mathrm{PI}(4,5) \mathrm{P}_{2}$ and $\mathrm{PI}(3,4,5) \mathrm{P}_{3}$, whereas hippocalcin showed somewhat weaker binding activity to these derivatives [11]. Notably, both proteins exhibit good binding capabilities for $\mathrm{PI}(4,5) \mathrm{P}_{2}$. 
Therefore, VILIP-1 seems to have a preference for highly phosphorylated PIs. The ability of hippocalcin to bind PI is not shared by VILIP-1.

\section{Co-localisation with phospholipids in hippocampal neurons}

To corroborate the in silico and in vitro findings, we looked at the interaction of VILIP-1 with $\mathrm{PI}(4,5) \mathrm{P}_{2}$ in hippocampal neurons. $\mathrm{PI}(4,5) \mathrm{P}_{2}$ is the most abundant phospho-inositide in cellular membranes. Immunofluorescence experiments evaluating the co-localisation of VILIP-1 with $\mathrm{PI}(4,5) \mathrm{P}_{2}$ (Fig. 5) revealed spatial vicinity of VILIP-1 and $\mathrm{PI}(4,5) \mathrm{P}_{2}$ at intracellular localisations and at defined spots at the cell surface (Fig. 5, co-localisation in yellow in C). These results further support the idea of direct interactions of VILIP-1 with PI(4,5) $\mathrm{P}_{2}$ and show localisation of VILIP-1 in defined signalling compartments at the cell surface, containing $\mathrm{PI}(4,5) \mathrm{P}_{2}$, a phospholipid involved in signal transduction processes.

\section{Conclusions}

Individual members of the family of NCS proteins localise to different subcellular membranes. Very recently, the occurrence of polybasic and polybasic-lipoyl motifs have been correlated with the targeting of plasma membrane proteins due to recognition of $\mathrm{PI}(3,4,5) \mathrm{P}_{3}$ and $\mathrm{PI}(4,5) \mathrm{P}_{2}[7 ; 8]$.

A similar observation is made with VILIPs and hippocalcin [11] which possess several basic residues in their $\mathrm{N}$-terminal region and are also $\mathrm{N}$-terminally myristoylated. Based on homology models, we hypothesised that inositol phosphate groups can be bound in the area between the Nterminal tail and the core of these proteins. Molecular dynamics simulations indicate that VILIP-1 is able to recognise various phospho-inositides derivatives in this area and that the anticipated basic amino acids play a key role in ligand binding. Using immobilised phospholipids, VILIP-1 was found to interact with the higher phosphorylated $\mathrm{PI}$ derivatives, such as $\mathrm{PI}(4,5) \mathrm{P}_{2}$ and $\mathrm{PI}(3,4,5) \mathrm{P}_{3}$. Results from monolayer adsorption experiments further support direct and specific interactions of VILIP-1 with PIP derivatives. The data on the subcellular localisation of VILIP-1 in hippocampal 
cells is in agreement with those findings, and puts further weight to the notion that interactions with specific PIPs may target VILIPs to distinct cellular signalling processes.

Our current working model assumes that VILIPs associate with the membrane via anchoring their myristoyl group in the bilayer and interact specifically with certain PIP derivatives via their conserved N-terminal residues. While further contributions to specificity by interactions with particular membrane receptors seem highly probable, PIP signalling may be a crucial component of the VILIP subcellular targeting mechanism.

With this study, we provide experimental evidence and testable hypotheses for molecular mechanisms of VILIP signalling. Further studies are under way in our laboratories to investigate the determinants of VILIP signalling in more detail and test the current working model.

\section{Experimental}

\section{Homology modelling and ligand structures}

The generation of homology models of VILIP-1 in the apo- and calcium-bound forms has been described elsewhere [10; 17]. Ligand structures mimicking the $\mathrm{PI}(4,5) \mathrm{P}_{2}$ and $\mathrm{PI}(3,4,5) \mathrm{P}_{3}$ head groups were constructed with the program SYBYL (Tripos). Since a study of VILIPs with membrane-bound PIPs was not attempted, the fatty acid chains were truncated resulting in glycerylphosphatidyl inositol derivatives $\mathrm{GI}(4,5) \mathrm{P}_{3}$ and $\mathrm{GI}(3,4,5) \mathrm{P}_{4}$ (see Fig. 1). The chiral centres of the structures were checked for correct conformation, charges of the ligand atoms were calculated and the geometry was optimised by energy minimisation using the SYBYL software.

\section{Manual ligand docking}

Initially, automated docking of $\mathrm{GI}(3,4,5) \mathrm{P}_{3}$ to VILIP-1 was attempted using the program FlexX running within the SYBYL software. The ligand was manually placed close to residues Lys28, Lys32 and Lys36, and a binding pocket around these residues was generated using a selection radius of $6.5 \AA$. Hydrogens were added and the ligand was docked using FlexX default parameters. 
Probably due to the spatial restrictions around the putative binding site in these models, this approach remained unsuccessful (data not shown).

In order to enable a fit of the ligand close to the lysine residues in the E1 helix, a slight rotation of about $2^{\circ}$ was performed between $\mathrm{C} \alpha(\mathrm{Thr} 20)$ and $\mathrm{C} \alpha(\mathrm{Glu} 21)$ using the graphics programme $\mathrm{O}$ [18]. Local refinement was carried out in $\mathrm{O}$ and the ligand was positioned manually with the phosphate groups being in approximate position to the lysine residues of helix E1.

\section{Molecular dynamics (MD) simulations}

MD simulations were carried out with Gromacs [19] following the tutorial by Kerrigan [20]. The Gromacs gmx force field and the spc water model were used. Ligand coordinate and topology files were obtained from the PRODRG server [21] using the PDB files from Sybyl (Tripos). To ensure a charge-neutral cell, sodium counter ions were added by replacing solvent molecules at sites of high electrostatic potential. A position-restrained dynamics simulation of 20 ps was performed to equilibrate the solvated protein-ligand complex and gradually heat the simulation cell to $300 \mathrm{~K}$. Periodic boundary conditions were applied in all three dimensions with the Particle Mesh Ewald (PME) method being used to treat the long-range electrostatic interactions. Trajectories were visualised using VMD [22] and further analyses were performed with Gromacs tools, Ligplot [23] and $\mathrm{O}$.

\section{Monolayer adsorption experiments}

Recombinant untagged VILIP-1 was bacterially expressed and purified by hydrophobic interaction chromatography (Hofmann et al., unpublished). Measurement of protein adsorption to phospholipid monolayers was carried out using a computer-controlled Langmuir film balance (NIMA Model $301 \mathrm{~A}$ ) at $20^{\circ} \mathrm{C}$. The area of the trough was $30 \mathrm{~cm} \mathrm{x} 5 \mathrm{~cm}$, and the instrument was equipped with a moveable barrier that allowed adjustment of the surface area of the monolayer. The subphase buffer containing $0.1 \mathrm{mM}$ EDTA, $100 \mathrm{mM} \mathrm{NaCl}, 20 \mathrm{mM}$ HEPES (pH 7.5) was filtered (0.1 mm), and 
poured into the trough until the surface was $2 \mathrm{~mm}$ higher than the trough brim (ca. $100 \mathrm{ml}$ ). The surface of the buffer was separated by the moveable barrier into two isolated areas. For optimal circulation and mixture, a small Teflon stirrer rotating at about $40 \mathrm{rpm}$, was placed in the compartment where no phospholipids were added. The surface pressure was measured with a surface potential meter using $1 \mathrm{~cm}$ x $2.3 \mathrm{~cm}$ plates cut from filter paper (Whatman, No. 1). The lipid solution was prepared as a mixture of DMPS and DMPC (3:1 molar ratio) dissolved in chloroform/methanol (2:1, v/v) at a concentration of $1 \mathrm{mg} / \mathrm{ml}$, and about $25 \mathrm{ml}$ of this solution was applied onto the surface of the subphase with a Hamilton syringe. Surface pressure-area isotherms were acquired in separate experiments before each protein adsorption experiment to determine the best volume of lipid suspension required to construct the monolayer. After spreading, the monolayer was left to equilibrate for $20 \mathrm{~min}$. The film was compressed by moving the barrier to generate a surface pressure $\mathrm{p}_{0}$ of about $15-17 \mathrm{mN} \mathrm{m}^{-1}$. The protein was injected into the subphase at a final concentration of $30 \mathrm{nM}$ using a Hamilton syringe extending beneath the barrier. The surface pressure $\pi$ was recorded as a function of time for about 45 min. Adsorption data were analysed with the software SDAR from the PCSB program collection [24].

In order to obtain monolayers with a conserved number of surface charges, the amount of PS component was adjusted in the case of the PIP-containing monolayers. When calculating the volumes of the lipid solution used for spreading, the amount of DMPS was decreased by 2 mol for each mol PI(4,5) $\mathrm{P}_{2 .}$, and by $3 \mathrm{~mol}$ for each mol PI(3,4,5) $\mathrm{P}_{3}$.

Since the commonly used assay control protein BSA is the principal transport protein of fatty acids in the cell plasma [25] and thus directly binds phospholipids [26], lysozyme has been chosen as a negative control for the adsorption experiments.

\section{Phospholipid overlay (PIP-strip) assay}

The purified protein was myristoylated as described elsewhere [27]. The GST-VILIP-1 fusion protein was prepared as described earlier [28]. For the PIP-strip assay, phospholipid membranes 
(Echelon, Salt Lake City, UT, U.S.A.) were blocked with 5\% skim milk powder and 2.5\% BSA in TBST for $3 \mathrm{~h}$ at room temperature. Prior to incubation with fusion proteins membranes were incubated with $1 \%$ BSA/TBST for $10 \mathrm{~min}$. Afterwards, nitrocellulose membranes were incubated with myristoylated VILIP-1, or as controls, GST-VILIP-1 fusion proteins or GST alone in the presence or absence of calcium ( $1 \mathrm{mM} \mathrm{CaCl}_{2}$ or $5 \mathrm{mM}$ EGTA) in $1 \% \mathrm{BSA} / \mathrm{TBST}$ at $4^{\circ} \mathrm{C}$ overnight. Unbound protein was washed away with TBST (three times for $10 \mathrm{~min}$ ). For the detection of bound protein, anti-VILIP-1 or anti-GST antibodies were used. Blots were incubated overnight at $4{ }^{\circ} \mathrm{C}$ with primary antibodies diluted in TRIS-buffered saline containing 0.2\% Tween-20 (TBST) and 5\% skim milk powder followed by incubation with appropriate secondary antibodies coupled to horseradish peroxidase. The immunoreactivity was visualised with the ECL detection system (Amersham).

\section{Hippocampal cell culture}

Hippocampal neurons in culture were prepared as previously described [29]. Briefly, hippocampi from fetal Wistar rat brains (embryonic day 18) were dissociated by enzyme digestion with $0.1 \%$ trypsin at $37^{\circ} \mathrm{C}$ for 20 min followed by titration through two different sized syringes. Cells were plated onto poly-D-lysin coated in 24 well culture plates with glass cover slips at a density of 60.000 cells in DMEM containing 10\% fetal calf serum, $2 \mathrm{mM} \mathrm{L-glutamine} \mathrm{and} \mathrm{antibiotics.} 24$ hours after plating culture medium was exchanged for neurobasal medium (Invitrogen) supplemented with 1x B27, $0.5 \mathrm{mM}$ L-glutamine and antibiotics and cells were maintained in a humidified $37^{\circ} \mathrm{C}$ atmosphere containing $5 \% \mathrm{CO}_{2}$.

\section{Immunocytochemistry}

Following fixation of hippocampal neurons (10-14 days in culture) with 3\% paraformaldehyde (PFA) in PBS, pH 7.4 for 5 min at room temperature, cells were washed twice with $25 \mathrm{mM}$ glycine in PBS to quench background staining. Subsequently the cells were blocked for surface staining in 
3\% bovine serum albumine, 10\% horse serum in PBS (blocking solution) or blocked and permeabilised with $0.1 \%$ Triton X-100 added to blocking solution for 30 min, respectively. Cells were incubated with primary antibodies (polyclonal rabbit anti-VILIP-1 antibody, anti$\mathrm{PI}(4,5) \mathrm{P}_{2}$ monoclonal IgG2b mouse antibody, Biomol) diluted in blocking solution at $4^{\circ} \mathrm{C}$ overnight. In background controls, the first antibodies were omitted. After washing three times with PBS secondary antibodies diluted in blocking solution without Triton X-100 were applied to the cells for $1 \mathrm{~h}$ at room temperature. After washing and mounting, Cy3 and Alexa Green 488 fluorescence were visualised using a laser scanning microscope (Leica DM 2500; Wetzlar, Germany). Images were recorded digitally and processed using Adobe Photoshop 7.0 (Adobe Systems, San Jose, CA).

\section{Acknowledgements}

We gratefully acknowledge funding by Fundacao Bial, the European League Against Rheumatism (EULAR) and the Rebecca L Cooper Foundation to AH, and funding by Deutsche Forschungsgemeinschaft to KHB (DFG Br-1579/8-1, Priority Program of the German Research Foundation, SPP1226 “Nicotine”, GRK 1123). Blessy Paul is recipient of a Griffith University International Research Scholarship. Wassim Altarche-Xifro is a qualifying student in the GRK programme 1123 of the DFG. 


\section{References}

[1] K. Braunewell, E. D. Gundelfinger, Cell Tissue Res. 1999, 295, 1.

[2] R. D. Burgoyne, D. W. O'Callaghan, B. Hasdemir, L. P. Haynes, A. V. Tepikin, Trends Neurosci. 2004, 27, 203.

[3] K. H. Braunewell, Trends Pharmacol. Sci. 2005, 26, 345.

[4] J. B. Ames, R. Ishima, T. Tanaka, J. I. Gordon, L. Stryer, M. Ikura, Nature 1997, 389, 198.

[5] J. B. Ames, T. Tanaka, L. Stryer, M. Ikura, Curr. Opin. Struct. Biol. 1996, 6, 432.

[6] M. R. Peitsch, S. McLaughlin, Biochemistry 1993, 32, 10436.

[7] W. D. Heo, T. Inoue, W. S. Park, M. L. Kim, B. O. Park, T. J. Wandless, T. Meyer, Science 2006, 314, 1458.

[8] M. P. Czech, Cell 2000, 100, 603.

[9] B. C. Lagerholm, G. E. Weinreb, K. Jacobson, N. L. Thompson, Annu. Rev. Phys. Chem. 2005, 56, 309 .

[10] K. H. Braunewell, M. Brackmann, A. Hofmann, Calcium Binding Proteins 2006, 1, 12.

[11] D. W. O'Callaghan, L. P. Haynes, R. D. Burgoyne, Biochem. J. 2005, 391, 231.

[12] C. Spilker, T. Dresbach, K. H. Braunewell, J. Neurosci. 2002, 22, 7331.

[13] C. Spilker, K. H. Braunewell, Mol. Cell. Neurosci. 2003, 24, 766.

[14] F. Blondeau, B. Ritter, P. D. Allaire, S. Wasiak, M. Girard, N. K. Hussain, A. Angers, V. Legendre-Guillemin, L. Roy, D. Boismenu et al., Proc. Natl. Acad. Sci. 2004, 101, 3833.

[15] M. A. De Matteis, A. Godi, Nat. Cell Biol. 2004, 6, 487.

[16] S. McLaughlin, D. Murray, Nature 2005, 438, 605.

[17] M. Brackmann, A. Hofmann, K. H. Braunewell, In: Neuronal calcium sensor proteins 2006 (Nova Science Publisher), p. 115.

[18] T. A. Jones, J. Y. Zou, S. Cowan, M. Kjeldgaard, Acta Crystallogr. A 1991, 47, 110.

[19] D. van der Spoel, E. Lindahl, B. Hess, G. Groenhof, A. E. Mark, H. J. C. Berendsen, J. Comp. Chem. 2005, 26, 1701.

[20] J. E. Kerrigan, http://www2.umdnj.edu/ kerrigje 2003

[21] A. W. Schuettelkopf, D. M. F. van Aalten, Acta Crystallogr. 2004, D60, 1355.

[22] W. Humphrey, A. Dalke, K. Schulten, J. Molec. Graphics 1996, 14, 33.

[23] A. C. Wallace, R. A. Laskowski, J. M. Thornton, Prot. Eng. 1995, 8, 127.

[24] A. Hofmann, A. Wlodawer, Bioinformatics 2002, 18, 209.

[25] J. Brown, P. Shockley, In: Lipid-protein interactions, vol. 11982 (John Wiley, New York), p. 25.

[26] E. J. F. Demant, G. V. Richieri, A. M. Kleinfeld, Biochem. J. 2002, 363, 809.

[27] I. Boekhoff, K. H. Braunewell, I. Andreini, H. Breer, E. Gundelfinger, Eur. J. Cell Biol. 1997, 72, 151.

[28] C. Spilker, K. Richter, K. H. Smalla, D. Manahan-Vaughan, E. D. Gundelfinger, K. H. Braunewell, Neuroscience 2000, 96, 121.

[29] M. Brackmann, S. Schuchmann, R. Anand, K. H. Braunewell, J. Cell Sci. 2005, 118, 2495. [30] M. J. Hartshorn, J. Comp. Aid. Mol. Des. 2002, 16, 871. 

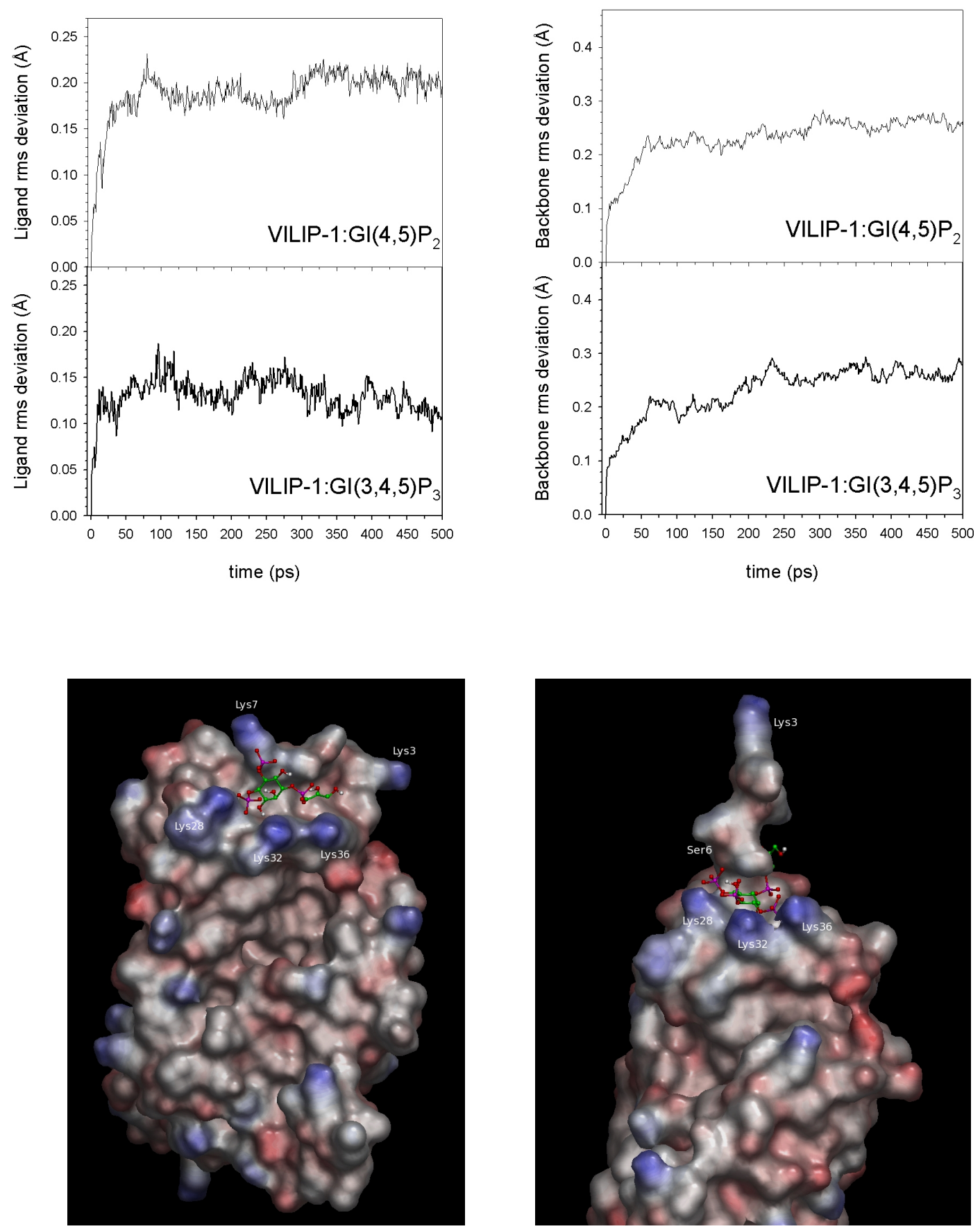

F ig. 1: Results from the molecular dynamics simulations.

Top left: Ligand rms deviation as a function of molecular simulation time. Top right: Protein backbone rms deviation as a function of molecular simulation time. Bottom: Orientation of $\mathrm{GI}(4,5) \mathrm{P}_{2}$ and $\mathrm{GI}(3,4,5) \mathrm{P}_{3}$ in the binding site of VILIP-1 after the molecular dynamics simulation. Picture prepared with AstexViewer [30]. 


\begin{tabular}{|c|c|}
\hline$(\mathrm{VILIP}-1)$ & $|---------|$ \\
\hline
\end{tabular}

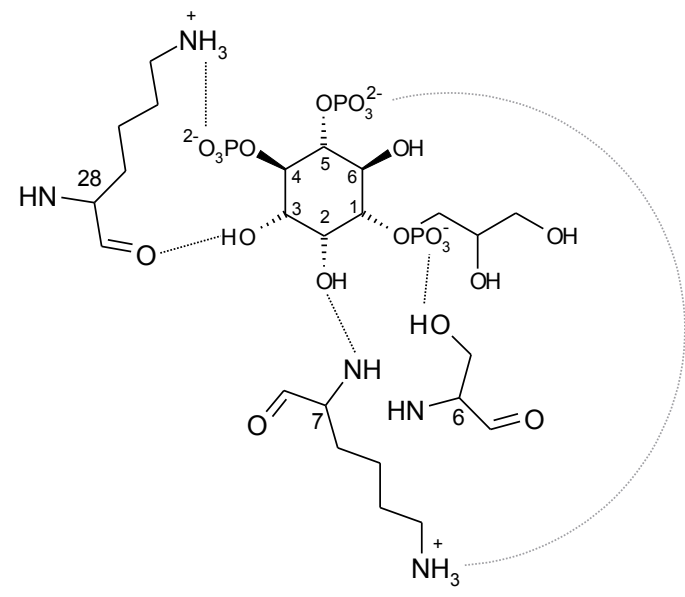

VILIP-1 : 4,5-GIP 3

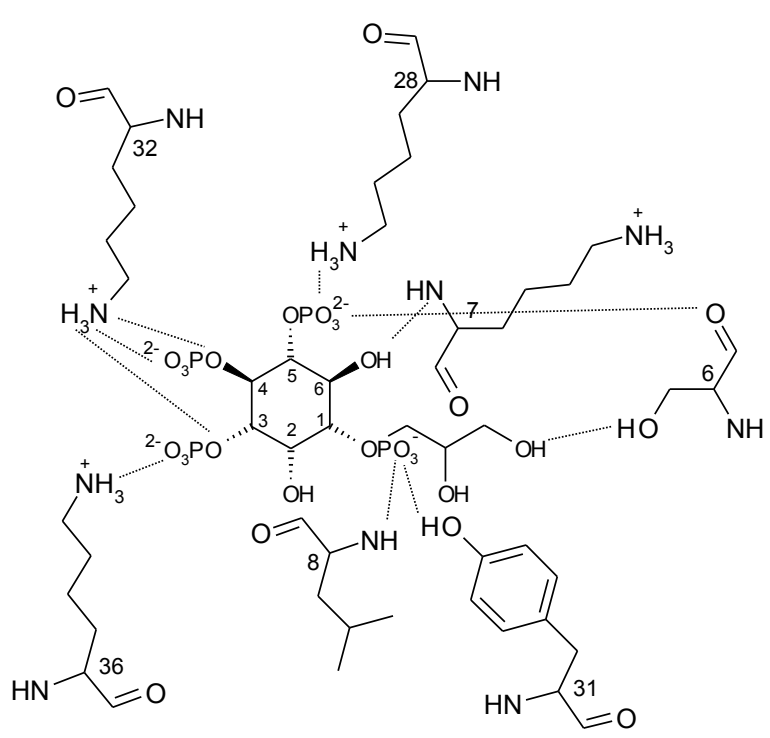

VILIP-1 : 3,4,5-GIP 4

F ig. 2: Protein-ligand interactions of VILIP-1:GIP complexes.

Top: Alignment of the N-terminal regions of NCS proteins. The conserved basic residues are highlighted in dark blue. Shown in light blue are residues found to interact with inositol phosphate derivatives as determined in this study. Bottom: The drawings show the interactions of $\mathrm{GI}(4,5) \mathrm{P}_{2}$ and $\mathrm{GI}(3,4,5) \mathrm{P}_{3}$ with VILIP-1 as obtained after docking and equilibration in molecular dynamics simulations. 
Figure 3

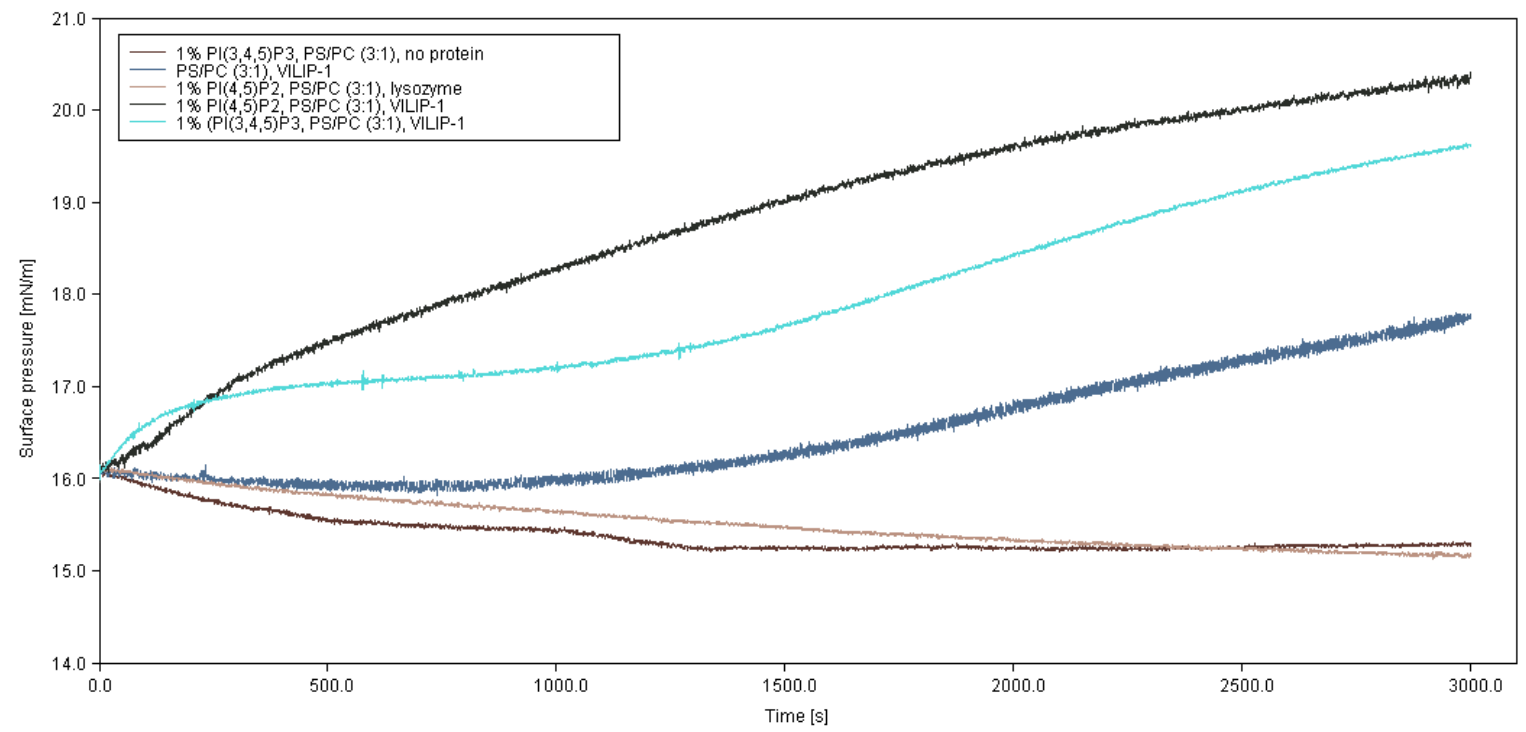

Fig. 3: Adsorption of unmyristoylated VILIP-1 to phospholipid monolayers in the absence of calcium.

VILIP-1 binding isotherms to a DMPS/DMPC(3:1) monolayer (dark blue), as well as monolayers with $1 \% \mathrm{PI}(4,5) \mathrm{P}_{2}$ (black) or PI( $\left.3,4,5\right) \mathrm{P}_{3}$ (light blue) are shown. The PIPcontaining monolayers have been constructed with charge conservation. Controls: Isotherm obtained after buffer injection into the subphase of a monolayer consisting of DMPS/DMPC(3:1) and $1 \% \mathrm{PI}(3,4,5) \mathrm{P}_{3}$ (dark brown); lysozyme isotherm with DMPS/DMPC(3:1) and 1\% PI(4,5) $\mathrm{P}_{2}$ (light brown). 
Figure 4
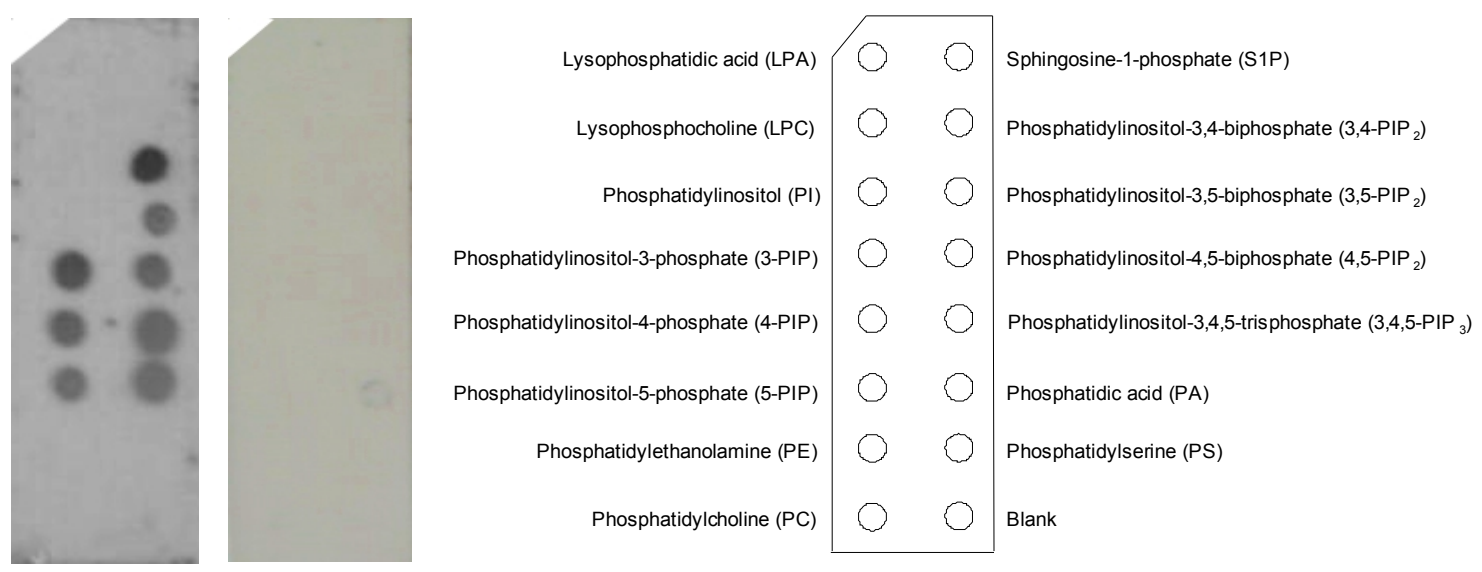

Fig. 4: VILIP-1 phospholipid overlay (PIP-strip) assays in the presence of calcium. Left: Results obtained with myristoylated untagged VILIP-1. Middle: Results obtained with unmyristoylated GST-VILIP-1. Right: The scheme shows the arrangement of different phospholipids on the strip. 
Figure 5

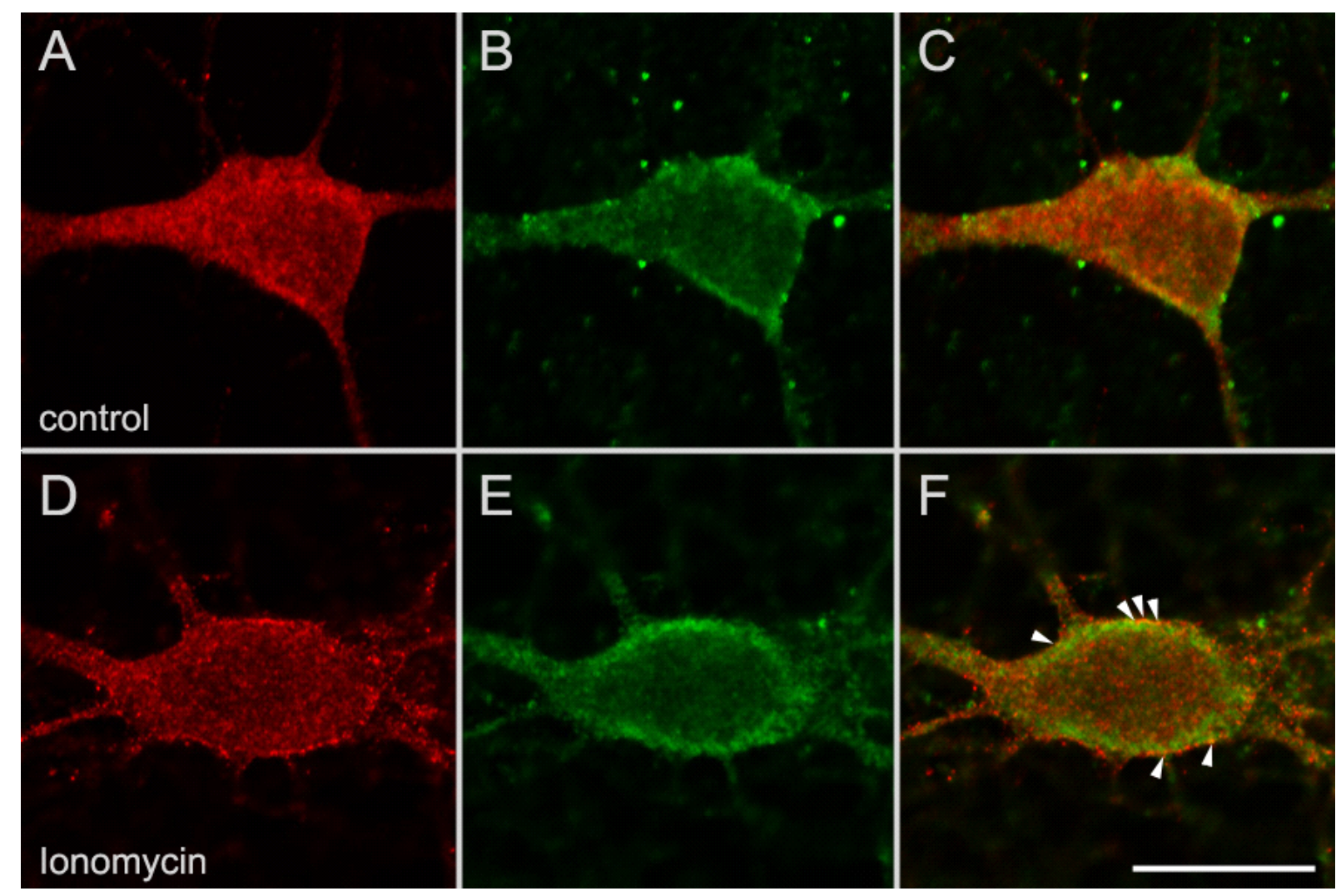

F ig. 5: Immunocytochemical co-localisation of VILIP-1 and PI $(4,5) \mathrm{P}_{2}$ in hippocampal neurons.

Co-localisation of VILIP-1 (A, $\boldsymbol{C}$, red channel) and $\mathrm{PI}(4,5) \mathrm{P}_{2}(\boldsymbol{B}, \boldsymbol{C}$, green channel $)$ was monitored by confocal immunofluorescence microscopy in hippocampal neurons after two weeks in culture. Co-localisation is shown as yellow (see arrows) in the merged image $(\boldsymbol{C})$. Bar in $\boldsymbol{C}$ is $20 \mu \mathrm{m}$. 